\title{
Particle production at energies available at the CERN Large Hadron Collider within an evolutionary model
}

\author{
Yu. M. Sinyukov ${ }^{1,2}$ and V. M. Shapoval ${ }^{1}$ \\ ${ }^{1}$ Bogolyubov Institute for Theoretical Physics, Metrolohichna 14b, Kiev 03680, Ukraine \\ ${ }^{2}$ Tomsk State University, Department of Physics, Lenin Avenue 36, Tomsk 634050, Russia
}

(Received 28 December 2017; published 4 June 2018)

\begin{abstract}
The particle yields and particle number ratios in $\mathrm{Pb}+\mathrm{Pb}$ collisions at the CERN Large Hadron Collider (LHC) energy $\sqrt{s_{N N}}=2.76 \mathrm{TeV}$ are described within the integrated hydrokinetic model (iHKM) at two different equations of state (EoS) for quark-gluon matter and the two corresponding hadronization temperatures $T=165 \mathrm{MeV}$ and $T=156 \mathrm{MeV}$. The role of particle interactions at the final afterburner stage of the collision in the particle production is investigated by means of comparison of the results of full iHKM simulations with those where the annihilation and other inelastic processes (except for resonance decays) are switched off after hadronization/particlization, similarly as in the thermal models. An analysis supports the picture of continuous chemical freeze-out in the sense that the corrections to the sudden chemical freeze-out results, which arise because of the inelastic reactions at the subsequent evolution times, are noticeable and improve the description of particle number ratios. An important observation is that, although the particle number ratios with switched-off inelastic reactions are quite different at different particlization temperatures which are adopted for different equations of state to reproduce experimental data, the complete iHKM calculations bring very close results in both cases.
\end{abstract}

DOI: 10.1103/PhysRevC.97.064901

\section{INTRODUCTION}

The analysis of the particle number ratios is carried out successfully in thermal models for different energies of $A+A$ collisions, from BNL Alternating Gradient Synchrotron (AGS) to CERN Large Hadron Collider (LHC) energies [1-7]. The thermal models suppose that, at some hypersurface characterized by uniform temperature and baryon chemical potential, the chemical composition of the hadron matter is frozen out, and in subsequent evolution of the hadron matter the particle yield is changed only because of the resonance decays. At LHC energies the afterburner "post-freeze-out" stage is the longest, and so there is a special interest to check the chemical freeze-out hypotheses within the dynamical models for these energies. The ALICE Collaboration has already noted $[8,9]$ that annihilation processes at the afterburner stage, which are taken into account in the hydrokinetic model (HKM) [10], noticeably improve agreement with (anti)proton spectra/yield at the LHC. The analysis of the role of inelastic processes at the post-hydrodynamic stage in formation of the particle yield is ongoing (see, e.g., [11]).

It seems that continuous chemical freeze-out as well as kinetic freeze-out is an inevitable feature of the dynamical models of $A+A$ collisions since sudden chemical freeze-

Published by the American Physical Society under the terms of the Creative Commons Attribution 4.0 International license. Further distribution of this work must maintain attribution to the author(s) and the published article's title, journal citation, and DOI. Funded by $S C O A P^{3}$. out means instant transition from extremely fast chemically equilibrated expansion (presupposing a very intensive inelastic reactions) to evolution with totally forbidden inelastic reactions. Sudden kinetic freeze-out means an instant change of hadron cross section from a very large one (typical for near perfect hydrodynamics) to zero cross section (free streaming particles). Such sudden transitions are not typical for realistic dynamical models. ${ }^{1}$ In our very recent note [12] we found, using a $K^{*}(892)$ probe, that at LHC energies a good agreement with the experimental data for these resonances requires a relatively long kinetic freeze-out, near $5 \mathrm{fm} / c$ after particlization/hadronization. It is worth noting that continuous thermal freeze-out means not only successive freeze-out for different hadrons (as in, e.g., Ref. [13]), but continuous particle emission for each species; see new important details in Ref. [12].

In this study we calculate the particle number ratios in the integrated hydrokinetic model (iHKM) and compare the results with the ones obtained in thermal models. Also we calculate the particle $p_{T}$ spectra in iHKM. We analyze the situation with different equations of state for quark-gluon and hadron matter and, correspondingly, at different temperatures of the so-called chemical freeze-out.

\section{MODEL DESCRIPTION}

The current study is carried out within the integrated hydrokinetic model (iHKM) [14] of relativistic nuclear collisions. This model includes the five stages of matter evolution and

\footnotetext{
${ }^{1}$ Note also that neither the first-order phase transition, nor the crossover are sudden in time in the process of system expansion.
} 
observable formation in $A+A$ collisions: the initial state formation, the prethermal matter evolution, the hydrodynamic stage, the particlization stage, and the hadronic cascade stage.

The initial energy-density profile in $\mathrm{iHKM}$ is associated with a quite early proper time, $\tau_{0} \approx 0.1 \mathrm{fm} / c$. According to a combined method, described in [14], one presents the generally nonequilibrium boost-invariant (in the central region of rapidity) parton/gluon distribution function on the initial hypersurface $\sigma_{0}: \tau=\tau_{0}$ in the factorized form

$$
f\left(t_{\sigma_{0}}, \mathbf{r}_{\sigma_{0}}, \mathbf{p}\right)=\epsilon\left(b ; \tau_{0}, \mathbf{r}_{T}\right) f_{0}(p),
$$

where $\epsilon\left(b ; \tau_{0}, \mathbf{r}_{T}\right)$, the initial energy density profile, is calculated in a hybrid approach, including both the wounded nucleon model and the binary collision approach. The proportion between the contributions of these two models to $\epsilon\left(b ; \tau_{0}, \mathbf{r}_{T}\right)$ is regulated by the parameter $0 \leqslant \alpha \leqslant 1$.

In iHKM simulations we obtain the distributions of numbers of wounded nucleons and binary collisions at $\tau_{0}$ with the help of the GLISSANDO code [15]. The weighed sum of such distributions (with the coefficients $\alpha$ and $1-\alpha$ ) is then multiplied by a normalizing factor $\epsilon_{0}$, the energy density at $\tau_{0}$ in the center of the system in central collisions. The value of $\epsilon_{0}$ is the main free parameter of the model, defined, together with the parameter $\alpha$, by means of fitting the observed mean charged particle multiplicity $d N_{c h} / d \eta$ dependence on centrality at a given collision energy. So, both $\epsilon_{0}$ and $\alpha$ parameters do not depend on collision centrality. However, changing the equation of state together with the corresponding particlization temperature will require a modification of $\epsilon_{0}$ and $\tau_{0}$ parameters. ${ }^{2}$ They are fixed in iHKM based on the measured multiplicity vs centrality distribution and the measured slope of the pion transverse momentum spectrum. As for the possible momentum anisotropy of the parton/gluon initial distribution, typical for the color-glass-condensate-based approaches, it is taken into account by the function $f_{0}(p)$ in Eq. (1), which is described in more detail in previous papers $[14,16]$ :

$$
f_{0}(p)=g \exp \left(-\sqrt{\frac{(p \cdot U)^{2}-(p \cdot V)^{2}}{\lambda_{\perp}^{2}}+\frac{(p \cdot V)^{2}}{\lambda_{\|}^{2}}}\right),
$$

where $\quad U^{\mu}=(\cosh \eta, 0,0, \sinh \eta) \quad$ and $\quad V^{\mu}=(\sinh \eta$, $0,0, \cosh \eta)$. In the rest frame of the fluid element one has $\eta=0,(p \cdot U)^{2}-(p \cdot V)^{2}=p_{\perp}^{2}$, and $(p \cdot V)^{2}=p_{\|}^{2}$, so that $\lambda_{\|}^{2}$ and $\lambda_{\perp}^{2}$ can be associated with the two effective temperatures: one along the beam axis and another along the axis orthogonal to it. In such a case the parameter $\Lambda=\lambda_{\perp} / \lambda_{\|}$ defines the momentum anisotropy of the initial state.

Once we have defined the initial conditions in the form of a nonthermal energy-momentum tensor, obtained from the distribution (1), we can proceed to the description of the prethermal matter dynamics, using the relaxation time approximation $[14,16,17]$. This pre-thermal stage starts in iHKM at the initial time $\tau_{0} \approx 0.1 \mathrm{fm} / c$, when the initial state is formed, and lasts till the thermalization time $\tau_{\text {th }}=1 \mathrm{fm} / c$,

\footnotetext{
${ }^{2}$ As for the parameter $\alpha$, in the current analysis it is not changed when switching to another EoS.
}

when an approximate local thermal equilibrium is supposed to be reached by the initially nonequilibrated system.

The subsequent matter evolution is described within a relativistic viscous hydrodynamics formalism, with the relativistic current and energy-momentum tensor in the Israel-Stewart form. We neglect there the bulk viscosity and heat conductivity terms. Since at the LHC energies the baryonic chemical potential in the spatiotemporal region, where the midrapidity observables are formed, is negligibly small, we put it to be just zero. According to the iHKM results [14] for identified hadron multiplicities, spectra, elliptic flow, and femtoscopy data, we put the minimal possible ratio of the shear viscosity coefficient to the entropy density, $\frac{\eta}{s}=\frac{1}{4 \pi}$ for the quark-gluon matter. The hydrodynamic approximation is justified as long as the matter can be considered remaining close to local chemical and thermal equilibrium. But at some temperature $T_{p}$ both such quasi-equilibrium descriptions get destroyed, and the system's further evolution should be described in terms of particles. A switching to such a description can be done either gradually or suddenly at the $T_{p}$ isotherm hypersurface. In this paper we utilize the latter mode of sharp particlization, comparing the simulation results in the cases of two different $T_{p}$ values. ${ }^{3}$ The construction and treatment of the particlization hypersurface in iHKM is realized through the Cornelius routine [18].

The last stage of the system's evolution within iHKM is a hadronic cascade stage, described with the help of the UrQMD model [19]. At this stage all the particles, previously produced at the particlization stage collide and interact with each other; that includes both elastic scatterings and inelastic processes, such as baryon-antibaryon annihilation. The unstable particles and resonance states decay (and recombine) at this stage as well.

\section{RESULTS AND DISCUSSION}

In the current paper we present the results for different particle number ratios and spectra, calculated in iHKM at two different particlization temperatures, $T_{p}=165 \mathrm{MeV}$ and $T_{p}=156 \mathrm{MeV}$, with two corresponding equations of state (EoS) for quark-gluon matter: the Laine-Schröder EoS [20] and the HotQCD Collaboration (HotQCD) EoS [21]. Using the two equations of state we investigate also whether the form of the EoS is significant for the description of particle number ratios in the evolutionary model with initial energy density $\epsilon\left(\tau_{0}\right)$ as a free parameter. Such a study is important since the extremely high rate of the fireball expansion, much larger than in the early Universe, would lead to modification of the effective equation of state as compared to the lattice QCD calculations for static system.

The ratios are calculated for central $(c=0-10 \%) \mathrm{Pb}+\mathrm{Pb}$ collisions at the LHC energy $\sqrt{s_{N N}}=2.76 \mathrm{TeV}$. The results for particle $p_{T}$ spectra are demonstrated for collisions with $c=0-5 \%$ and serve as an additional justification of the choice of model parameters (which do not depend on centrality).

\footnotetext{
${ }^{3}$ In fact, we suppose that the particlization temperature $T_{p}$ coincides with the temperature when the hadronization process is (almost) completed.
} 


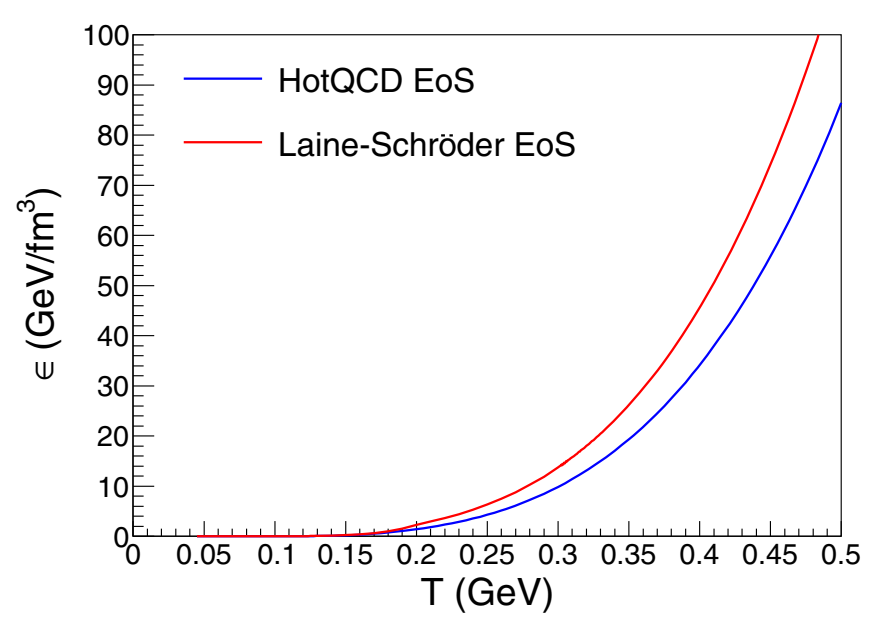

FIG. 1. A comparison of two equations of state for quark-gluon matter: the Laine-Schröder EoS [20], corresponding to the particlization temperature $T_{p}=165 \mathrm{MeV}$, and the HotQCD Collaboration "HotQCD" EoS [21], corresponding to the particlization temperature $T_{p}=156 \mathrm{MeV}$.

The Laine-Schröder equation of state was previously used in HKM, the predecessor of $\mathrm{iHKM}$, as the lattice QCD inspired EoS, ensuring that the description of dense quarkgluon matter and its crossover-type transition to the hadron resonance gas pass without gaps in pressure and energy density. The corresponding particlization temperature $T_{p}=165 \mathrm{MeV}$ was used in HKM calculations, that resulted in a successful simultaneous description of a variety of observables in heavy ion collision experiments at RHIC and LHC (spectra, interferometry radii, $v_{2}$ coefficients, source functions, etc. [10,2225]). The "HotQCD" EoS corresponds to the recent HotQCD Collaboration results from lattice QCD simulations devoted to the quark-gluon matter state description. The respective particlization temperature, $T_{p}=156 \mathrm{MeV}$, is in agreement with the most recent estimates of the chemical freeze-out temperature obtained in the thermal model, $T_{c h}=156 \pm 1.5$ $\mathrm{MeV}$ [26]. In Fig. 1 one can see a comparison of the two EoS on the plot in the coordinates $(\epsilon, T)$. The Laine-Schröder EoS corresponds to more rapidly growing energy density at high temperatures.

The iHKM parameter values, used in the analysis in the case of $T_{p}=165 \mathrm{MeV}$, are chosen to be the same as those that have provided the optimal description of multiple LHC bulk observables [16]: $\tau_{0}=0.1 \mathrm{fm} / c, \tau_{\text {th }}=1 \mathrm{fm} / c$, the relaxation time at the pre-thermal stage $\tau_{\text {rel }}=0.25 \mathrm{fm} / c, \epsilon_{0}=680$ $\mathrm{GeV} / \mathrm{fm}^{3}, \alpha=0.24$, and the momentum anisotropy of the initial state $\Lambda=100$. For the new particlization temperature $T_{p}=156 \mathrm{MeV}$ most parameter values remain the same, except for $\epsilon_{0}=495 \mathrm{GeV} / \mathrm{fm}^{3}$ and $\tau_{0}=0.15 \mathrm{fm} / c$, which are changed in order to ensure the correct charged particle multiplicity and pion $p_{T}$ spectrum slope.

In Fig. 2 one can see a comparison of transverse momentum spectra calculated in iHKM for $c=0-5 \% \mathrm{~Pb}+\mathrm{Pb}$ collisions in the two mentioned regimes $\left(T_{p}=165 \mathrm{MeV}\right.$ and $T_{p}=156 \mathrm{MeV}$ ) together with the experimental points. At both particlizaton temperatures the model gives a sufficiently

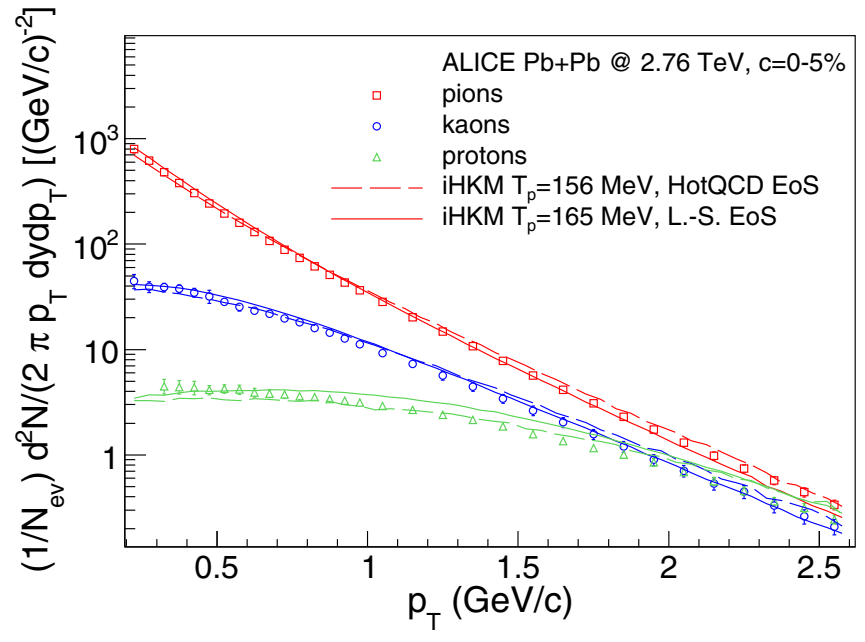

FIG. 2. The pion, kaon, and proton $p_{T}$ spectra calculated in iHKM at two particlization temperatures, $T_{p}=165 \mathrm{MeV}$ and $T_{p}=156 \mathrm{MeV}$ and corresponding equations of state [20] and [21], compared with the ALICE experimental data [9] for central $(c=0-5 \%) \mathrm{Pb}+\mathrm{Pb}$ collisions at the LHC energy $\sqrt{s_{N N}}=2.76 \mathrm{TeV}$.

good description of the data, which confirms that the model parameters are chosen correctly.

In Figs. 3 and 4 we demonstrate the iHKM results for a set of particle number ratios and compare them with the experimental results and those obtained from the thermal model [26,27]. Here the iHKM simulations are performed in two regimes: full calculation and the mode with the inelastic processes switched off (except for resonance decays). It is worth noting that the calculations without inelastic reactions, but with the initial conditions adjusted to provide the right description

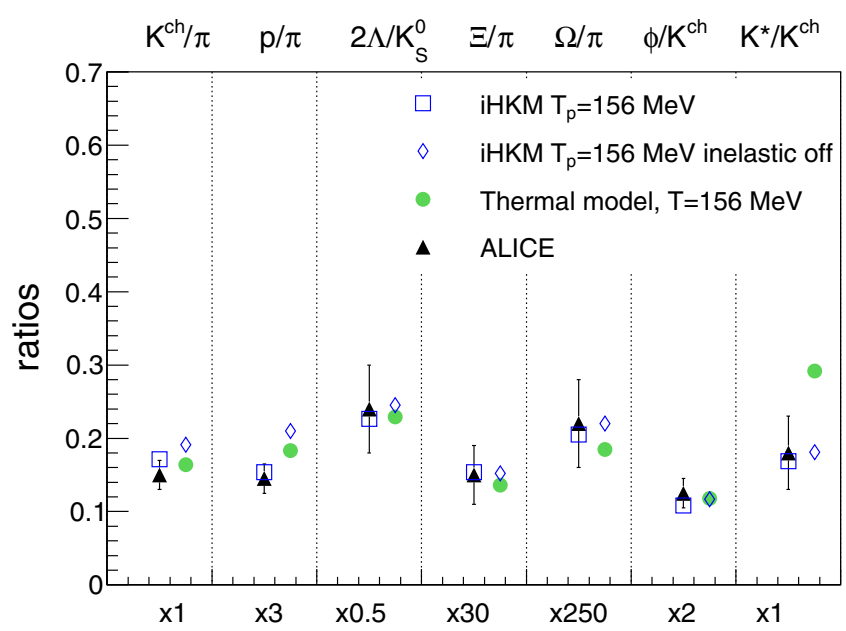

FIG. 3. A comparison of particle number ratios, calculated in iHKM (blue markers) at the particlization temperature $T_{p}=156 \mathrm{MeV}$ and the HotQCD Collaboration equation of state, with the ALICE experimental data [28] and the thermal model results at $T=156$ $\mathrm{MeV}$ [26]. The iHKM simulations are performed in two regimes: full calculation and the mode with inelastic reactions (except for resonance decays) switched off. The $\chi^{2}$ values for these two regimes are 2.2 and 14.9 respectively. 


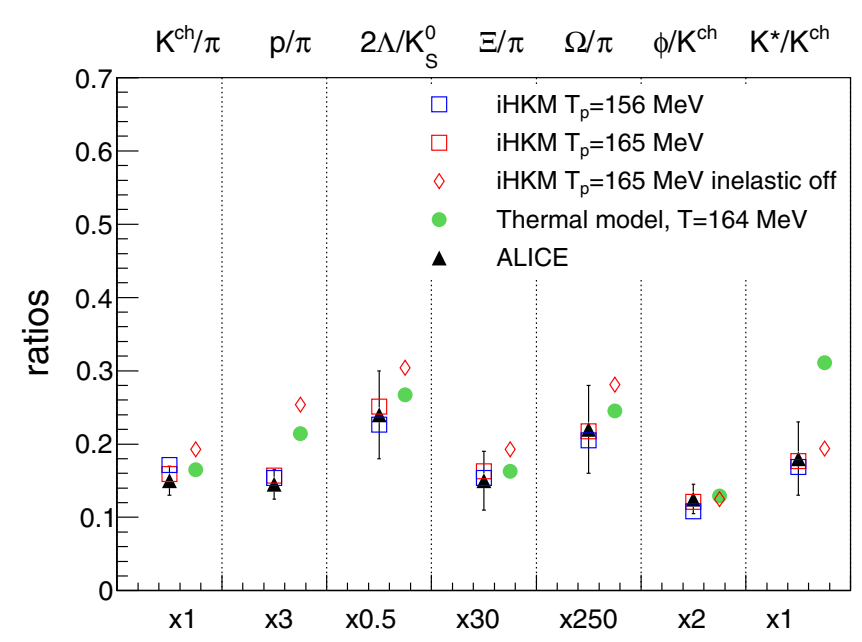

FIG. 4. The same as in Fig. 3, but the results for the iHKM calculation at $T_{p}=165 \mathrm{MeV}$ and the Laine-Schröder equation of state are shown. The thermal model results are demonstrated for $T=164 \mathrm{MeV}[26,27]$. The $\chi^{2}$ values for the full and "switchedoff-inelastic" iHKM simulations are 0.7 and 37.7 respectively.

of the charged hadron multiplicities, give the same particle number ratios as without such an adjustment. This effect is clear: when we switch off the inelastic reactions (except for the resonance decays), then all the particle numbers on the hypersurface of the chemical freeze-out are proportional to the effective volume, $N_{i}=n_{i}(T, \mu) V_{\text {eff }}$, [29], which absorbs the hydro-velocities and space-time characteristics at the chemical freeze-out: $V_{\text {eff }}=\int_{\sigma_{c h}} u^{\mu}(x) d \sigma_{\mu}(x)$. The same happens with the similarly defined effective volume, related to the unity of rapidity in the case of boost invariance in the midrapidity region [29]. Therefore, when one fits the initial energy density (and the related initial time) in order to adjust multiplicity distribution at the artificially truncated "switched-off-inelastic" dynamics at the afterburner stage, the only common factor $V_{\text {eff }}$ will be modified (EoS and corresponding particlization temperature are fixed). So, the particle number ratios will not change, no matter whether the initial conditions are retuned or not.

As one can see from the figures, the thermal model and the iHKM results, related to the case when the inelastic scatterings are switched off, ${ }^{4}$ are modified noticeably when the temperature $T_{p}$ (or $T_{\mathrm{ch}}$ in the thermal models) is changing,

\footnotetext{
${ }^{4}$ Note that some deviation of iHKM results in this truncated case from those of the thermal model should be connected with the number of resonances taken into account. In the iHKM case we consider 329 types of resonances. As for the large deviation in the case of the $K^{*} / K^{c h}$ ratio, it can be explained by different definitions of the $K^{*} / K^{c h}$ ratio in the experiment and iHKM from the one side and the thermal model calculations from the other side. As follows from the experimental papers, e.g., [30], the $K^{* 0}(892)$ resonances are reconstructed via the products of their decay into $K^{+} \pi^{-}$pairs with branching ratio 0.66 (while the $K^{* 0}$ 's decaying through a channel $K^{* 0} \rightarrow K^{0} \pi^{0}$ are excluded from the analysis). The same reconstruction procedure is applied in the iHKM study. Hence, the number of $K^{*}$ 's identified in such a way is about $2 / 3$ of the full $K^{*}$
}

and describe the data worse than the full iHKM calculations. As for the latter, they give very close results at both particlization temperatures and equations of state.

In a very recent paper [12] an essential influence of the particle rescatterings at the afterburner stage of the collision on the $K^{*}(892)$ resonance observability was shown. It means that the so-called thermal freeze-out is not sharp or sudden, but continuous. Our current study points out the dynamical continuous character of the so-called chemical freeze-out in relativistic heavy ion collisions. It demonstrates that accounting for inelastic processes at the afterburner stage of the collision plays a more important role in the correct description of experimental observables than the specific choice of the supposed particlization/hadronization temperature.

\section{CONCLUSIONS}

The particle $p_{T}$ spectra and particle number ratios calculated in iHKM with two different thermodynamic equations of state and corresponding particlization/hadronization temperatures demonstrate that a satisfactory description of the experimental data can be achieved at both $T_{p}$ values if the initial energy density $\epsilon\left(\tau_{0}\right)$ is the free parameter. In this sense the results practically do not depend on the equation of state in complete dynamics of rapidly expanding fireballs formed in $A+A$ collision. However, the situation is different when one truncates the post-hydrodynamic stage: the description is better for lower temperature of chemical freeze-out, $T=$ $156 \mathrm{MeV}$. But even in this case-when annihilation and other inelastic processes (except for the resonance decays) at the afterburner stage are neglected-the theoretical results get worse as compared to the full calculations. One can conclude that neither thermal nor chemical freeze-out can be considered to be sudden at some corresponding temperatures. Our analysis shows that even at the minimal hadronization temperature near $155 \mathrm{MeV}$, the annihilation and other nonelastic scattering reactions still play noticeable roles in the formation of particle number ratios, especially those where protons and pions are participating.

The fact that the results of the iHKM evolutionary model for small and relatively large particlization temperatures are quite similar means that inelastic processes (other than the resonance decays), which occur during the matter evolution below the corresponding temperature, play the role of a compensatory mechanism in formation of the particle number ratios.

Thus, the current analysis supports the picture of continuous chemical freeze-out at the LHC in the sense that the corrections to the sudden chemical freeze-out results, accounting for the inelastic reactions at the subsequent times, are important and improve the description of the experimental data.

\section{ACKNOWLEDGMENTS}

Yu.M. thanks P. Braun-Munzinger for fruitful and stimulating discussions. The research was carried out within the scope

number. In contrast, the thermal model describes the full $K^{*}$ number and therefore gives a higher $K^{*} / K^{c h}$ ratio. 
of the EUREA: European Ultra Relativistic Energies Agreement (European Research Group: "Heavy ions at ultrarelativistic energies"), Agreement F-2018 with the National Academy of Sciences (NAS) of Ukraine. The work is partially supported by the Tomsk State University Competitiveness Improvement Program (project 5-100), and the Targeted research program of the NAS of Ukraine "Fundamental research on high-energy physics and nuclear physics (international cooperation)."
[1] P. Braun-Munzinger, J. Stachel, J. P. Wessels, and N. Xu, Phys. Lett. B 344, 43 (1995).

[2] P. Braun-Munzinger, J. Stachel, J. P. Wessels, and N. Xu, Phys. Lett. B 365, 1 (1996).

[3] P. Braun-Munzinger, I. Heppe, and J. Stachel, Phys. Lett. B 465, 15 (1999).

[4] F. Becattini, M. Gazdzicki, and J. Sollfrank, Eur. J. Phys. C 5, 143 (1998).

[5] J. Cleymans and K. Redlich, Phys. Rev. C 60, 054908 (1999).

[6] P. Braun-Munzinger, D. Magestro, K. Redlich, and J. Stachel, Phys. Lett. B 518, 41 (2001).

[7] P. Braun-Munzinger, V. Koch, T. Schafer, and J. Stachel, Phys. Rep. 621, 76 (2016).

[8] B. Abelev et al. (ALICE Collaboration), Phys. Rev. Lett. 109, 252301 (2012).

[9] B. Abelev et al. (ALICE Collaboration), Phys. Rev. C 88, 044910 (2013).

[10] Iu. A. Karpenko, Yu. M. Sinyukov, and K. Werner, Phys. Rev. C 87, 024914 (2013).

[11] F. Becattini et al., in New Horizons in Fundamental Physics (Springer, Berlin, 2016), pp. 139-150.

[12] V. M. Shapoval, P. Braun-Munzinger, and Yu. M. Sinyukov, Nucl. Phys. A 968, 391 (2017).

[13] S. Chatterjee, R. M. Godbole, and S. Gupta, Phys. Lett. B 727, 554 (2013).

[14] V. Yu. Naboka, Iu. A. Karpenko, and Yu. M. Sinyukov, Phys. Rev. C 93, 024902 (2016).

[15] W. Broniowski, M. Rybczynski, and P. Bozek, Comput. Phys. Commun. 180, 69 (2009).

[16] V. Yu. Naboka, S. V. Akkelin, Iu. A. Karpenko, and Yu. M. Sinyukov, Phys. Rev. C 91, 014906 (2015).
[17] S. V. Akkelin and Yu. M. Sinyukov, Phys. Rev. C 81, 064901 (2010).

[18] P. Huovinen and H. Petersen, Eur. Phys. J. A 48, 171 (2012); S. Pratt, Phys. Rev. C 89, 024910 (2014); D. Molnar and Z. Wolff, ibid. 95, 024903 (2017).

[19] S. A. Bass et al., Prog. Part. Nucl. Phys. 41, 255 (1998); M. Bleicher et al., J. Phys. G 25, 1859 (1999).

[20] M. Laine and Y. Schröder, Phys. Rev. D 73, 085009 (2006).

[21] A. Bazavov, T. Bhattacharya, C. DeTar, H. T. Ding, S. Gottlieb, R. Gupta, P. Hegde, U. M. Heller, F. Karsch, E. Laermann, L. Levkova, S. Mukherjee, P. Petreczky, C. Schmidt, C. Schroeder, R. A. Soltz, W. Soeldner, R. Sugar, M. Wagner, and P. Vranas (The HotQCD Collaboration), Phys. Rev. D 90, 094503 (2014).

[22] V. M. Shapoval, P. Braun-Munzinger, Iu. A. Karpenko, and Yu. M. Sinyukov, Nucl. Phys. A 929, 1 (2014).

[23] V. M. Shapoval, P. Braun-Munzinger, Iu. A. Karpenko, and Yu. M. Sinyukov, Phys. Lett. B 725, 139 (2013).

[24] V. M. Shapoval, Yu. M. Sinyukov, and Iu. A. Karpenko, Phys. Rev. C 88, 064904 (2013).

[25] Yu. M. Sinyukov, V. M. Shapoval, and V. Yu. Naboka, Nucl. Phys. A 946, 227 (2016).

[26] J. Stachel, A. Andronic, P. Braun-Munzinger, and K. Redlich, J. Phys. Conf. Ser. 509, 012019 (2014).

[27] A. Andronic et al., J. Phys. G: Nucl. Part. Phys. 38, 124081 (2011).

[28] M. Floris, Nucl. Phys. A 931, 103 (2014).

[29] S. V. Akkelin, P. Braun-Munzinger, and Yu. M. Sinyukov, Nucl. Phys. A 710, 439 (2002).

[30] B. B. Abelev et al., (The ALICE Collaboration), Phys. Rev. C 91, 024609 (2015). 